Highly Energetic Physical Processes and

Mechanisms for Emission from Astrophysical Plasmas

IAU Symposium, Vol. 195, 2000

P. C. H. Martens, S. Tsuruta, and M. A. Weber, eds.

\title{
Compton Scattering, Pair Annihilation, and Pair Production in a Plasma
}

\author{
V. Krishan \\ Indian Institute of Astrophysics, Bangalore 560034, India
}

\begin{abstract}
The square of the four-momentum of a photon in vacuum is zero. However, in an unmagnetized plasma, it is equal to the square of the plasma frequency. Further, the electron-photon coupling vertex is modified in a plasma to include the effect of the plasma medium. I calculate the cross sections of three processes in a plasma-Compton scattering and electron-positron pair annihilation and production. At high plasma densities, the cross sections are found to change significantly. Such high plasma densities exist in several astrophysical sources.
\end{abstract}

\section{Introduction}

The processes of Compton scattering and electron-positron pair annihilation and production play an important role in the functioning of highly energetic astrophysical sources such as pulsars, supernovae, $\gamma$-ray bursts, and the early universe (Wolfgang, Fabian, \& Giovannelli 1990; Rose 1973). The cross sections of these processes are used, almost always, either in the classical Thomson limit or, at best, including the Klein-Nishina corrections for high-energy photons. To the best of my knowledge, the effect of the plasma medium on the cross sections has not been investigated. In this paper, I show that the cross sections change significantly when the presence of the plasma medium is taken into account. The plasma medium affects in two ways. First, the square of the four-momentum of a photon is no longer zero, it is equal to the square of the plasma frequency; and second, the electron-photon vertex now depends upon the dielectric constant $\epsilon$ of the plasma medium. The cross sections become functions of the plasma density $n$.

The dispersion relation of photons in an unmagnetized plasma is given by $\omega^{2}=\omega_{p}^{2}+\vec{k}^{2} c^{2}$, where $\omega$ is the photon frequency, $\vec{k}$ is the photon wavevector, and the plasma frequency $\omega_{p}=\left(4 \pi n e^{2} / m\right)^{1 / 2}$ in an electron-proton plasma and $=\left(8 \pi n e^{2} / m\right)^{1 / 2}$ in an electron-positron plasma of density $n$. Thus, the square $k^{2}$ of the photon four-momentum $k=(\omega, \vec{k} c)$ is found to be $\omega^{2}-k^{2} c^{2}=\omega_{p}^{2}$ (Krishan 1999). The total energy associated with a wave in a dielectric (Harris 1975; Landau \& Lifshitz 1960) is modified by the Von Laue factor $\frac{1}{2 \omega} \frac{\partial}{\partial \omega}\left(\omega^{2} \epsilon(\omega)\right)$ so that the vector potential $\vec{A}(x)$ is defined as: 


$$
\vec{A}(x)=\left[\frac{2 \pi \hbar c^{2}}{V\left[\frac{1}{2} \frac{\partial}{\partial \omega} \omega^{2} \epsilon\right]_{\omega_{k}}}\right]^{1 / 2} \Lambda_{\vec{k} \sigma}\left[a_{\vec{k} \sigma} e^{i k \cdot x}+a_{\vec{k} \sigma}^{\dagger} e^{-i k \cdot x}\right]
$$

where the $a$ 's are the photon creation and annihilation operators. For an unmagnetized plasma, the dielectric function $\epsilon=1-\omega_{p}^{2} / \omega^{2}$. The vector potential $\vec{A}(\vec{x})$ is modified only to the extent that the photon dispersion relation in vacuum is replaced by the photon dispersion relation in a plasma. With these changes, one can proceed to calculate the cross sections for the three processes, related by crossing symmetry, using the standard techniques of quantum electrodynamics (Reinhardt 1992, 1994).
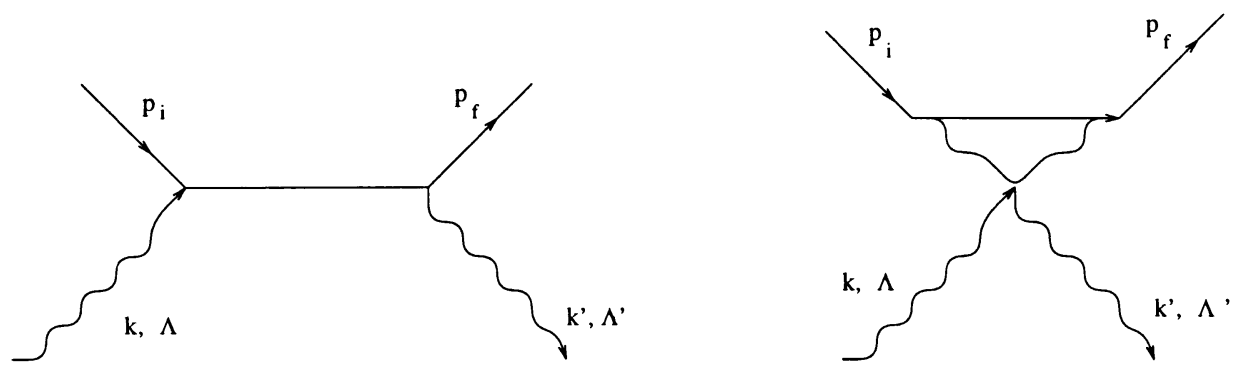

Figure 1. The direct and exchange diagrams for Compton scattering: $e^{-}+\gamma \rightarrow \gamma^{\prime}+e^{-}$.

\section{Compton Scattering}

Compton scattering consists of the absorption of an incoming photon with fourmomentum $k$ and polarization vector $\Lambda$ by an electron, with the emission of a second photon with four-momentum $k^{\prime}$ and polarization vector $\Lambda^{\prime}$. The Feynman diagrams for this process are shown in Figure 1. Here, $p_{i}$ and $p_{f}$ are the fourmomenta of the initial and final electrons, respectively.

The differential cross section for the Compton scattering averaged over photon polarizations is found to be

$$
\begin{array}{r}
\frac{\mathrm{d} \bar{\sigma}_{\mathrm{c}}}{\mathrm{d} \Omega_{\mathrm{k}}} \frac{1}{r_{0}^{2}}=\left[\frac{\gamma^{\prime 2}}{\gamma^{2}} \frac{\left(1+\cos ^{2} \theta\right)}{2 \sqrt{A B}}+\frac{\gamma^{\prime 2}}{4 \gamma^{2}}\left[\frac{\gamma^{\prime}}{\gamma A}+\frac{\gamma}{\gamma^{\prime} B}-\frac{2}{\sqrt{A B}}+\right.\right. \\
+\left(1-\frac{\sqrt{A}}{\sqrt{B}}\right) \frac{\gamma}{A}(1-p) \sin ^{2} \theta-\left(1-\frac{\sqrt{B}}{\sqrt{A}}\right) \frac{\gamma^{\prime}}{B}\left(1-p^{\prime}\right) \sin ^{2} \theta- \\
\left.\left.-\frac{p \gamma}{A B}\left\{\frac{2 \gamma}{\gamma^{\prime}} \sqrt{A B}+A\left(\frac{\gamma^{2}}{\gamma^{\prime 2}}+\frac{\gamma}{\gamma^{\prime}}\right)-B\left(\frac{\gamma^{\prime}}{\gamma}+1\right)-2 \sqrt{A B}\right\}\right]\right] G
\end{array}
$$



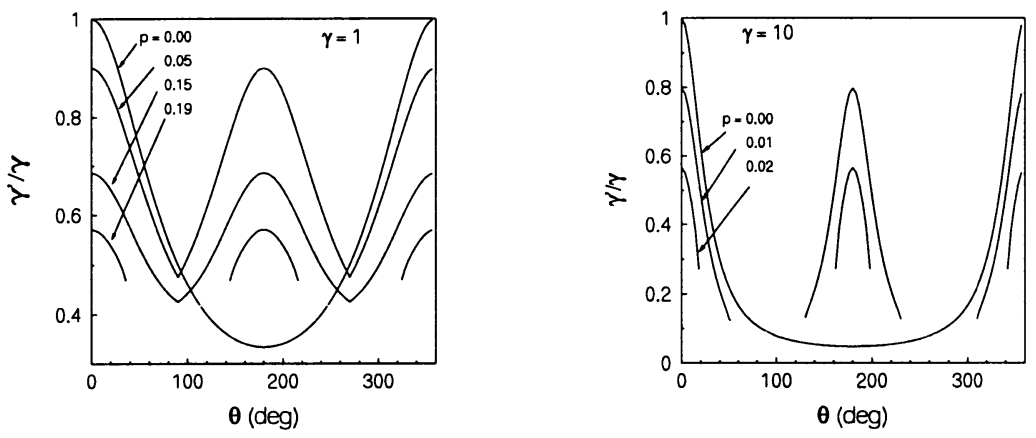

Figure 2. Variation of the first root $\left(\gamma^{\prime} / \gamma\right)$ of Eqn. (3) vs. the scattering angle $\theta$ for different values of the plasma density parameter $p$ and initial photon energy $\gamma$.
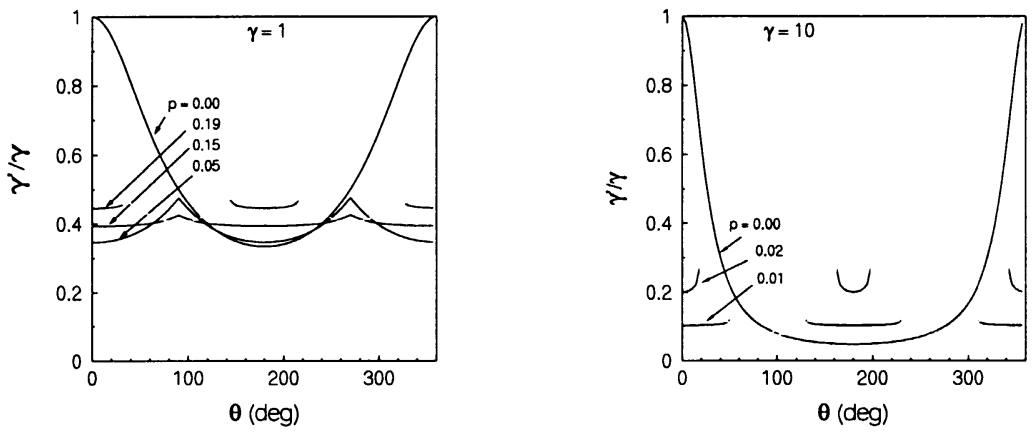

Figure 3. Variation of the second root $\left(\gamma^{\prime} / \gamma\right)$ of Eqn. (3) vs. the scattering angle $\theta$ for different values of the plasma density parameter $p$ and initial photon energy $\gamma$.
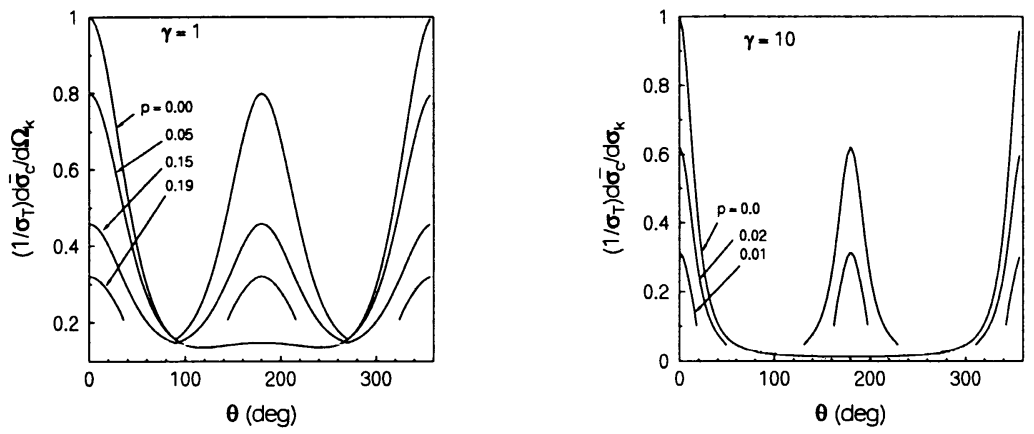

Figure 4. Variation of the differential cross section for Compton scattering with the scattering angle $\theta$ for different values of the plasma density parameter $p$, the initial photon energy $\gamma$, and the final photon energy $\gamma^{\prime}$ corresponding to the first root of Eqn. (3). 

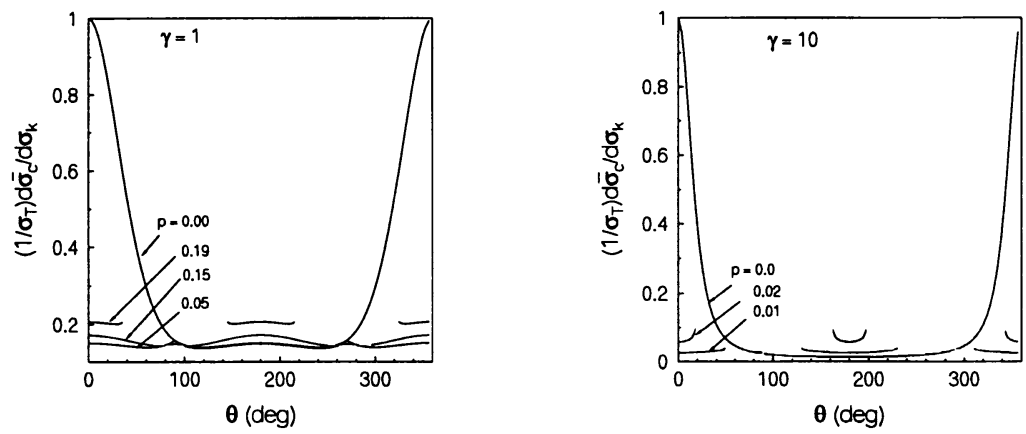

Figure 5. Variation of the differential cross section for Compton scattering with the scattering angle $\theta$ for different values of the plasma density parameter $p$, the initial photon energy $\gamma$, and the final photon energy $\gamma^{\prime}$ corresponding to the second root of Eqn. (3).
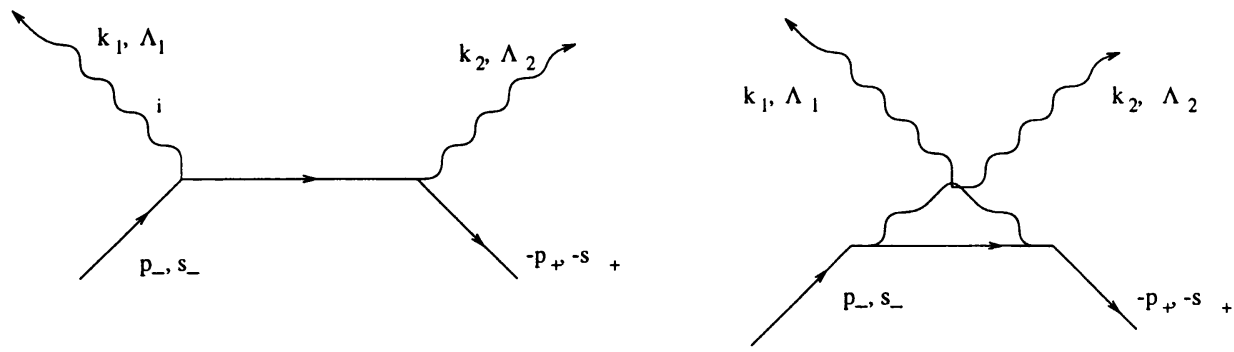

Figure 6. Direct and exchange diagrams for electron-positron annihilation : $e^{-}+e^{+} \rightarrow \gamma_{1}+\gamma_{2}$.

where $\gamma=\hbar \omega / m c^{2}$ and $\gamma^{\prime}=\hbar \omega^{\prime} / m c^{2}$ are the initial and final photon energies related by the energy-momentum conservation law,

$$
p+\frac{1}{\gamma}=\gamma^{\prime} / \gamma^{2}+\left(\gamma^{\prime} / \gamma\right)\left\{1-\sqrt{1-p} \sqrt{1-p^{\prime}} \cos \theta\right\}
$$

and

$$
G=\left[1+p \gamma+p^{\prime} \gamma^{\prime} \frac{\sqrt{1-p}}{\sqrt{1-p^{\prime}}} \cos \theta\right]^{-1} \sqrt{1-p^{\prime}} .
$$

Here, $\theta$ is the scattering angle, $A=(1+p \gamma / 2)^{2}, B=\left(1-p^{\prime} \gamma^{\prime} / 2\right)^{2}, p=\omega_{p}^{2} / \omega^{2}$, $p^{\prime}=\omega_{p}^{2} / \omega^{\prime 2}$, and $r_{0}^{2}=\left(e^{2} / m c^{2}\right)^{2}$. One can easily identify the Thomson, KleinNishina, and additional terms which depend upon the plasma frequency. Unlike in vacuum, the initial and final photon frequencies are related through a quadratic expression. For every incoming photon, there are two possible scattered photons. The two values of the ratio $\left(\gamma^{\prime} / \gamma\right)$ are plotted against the scattering angle $\theta$ for various values of $p$ and $\gamma$ in Figures 2 and 3 . The first root 

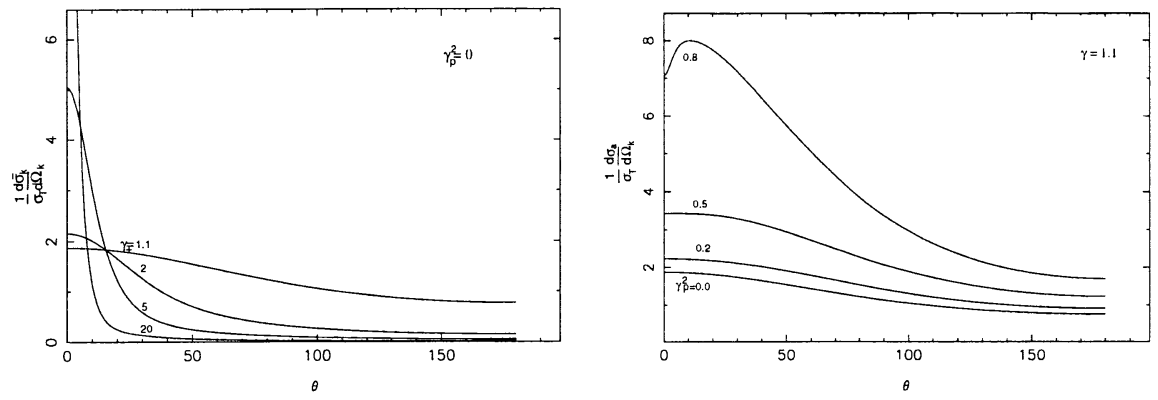

Figure 7. Variation of the differential cross section for pair annihilation with the angle $\theta$ between the positron beam and the photon $\left(\gamma_{1}\right)$ for different positron energies $\gamma_{+}$and plasma density parameters $\gamma_{p}^{2}$.
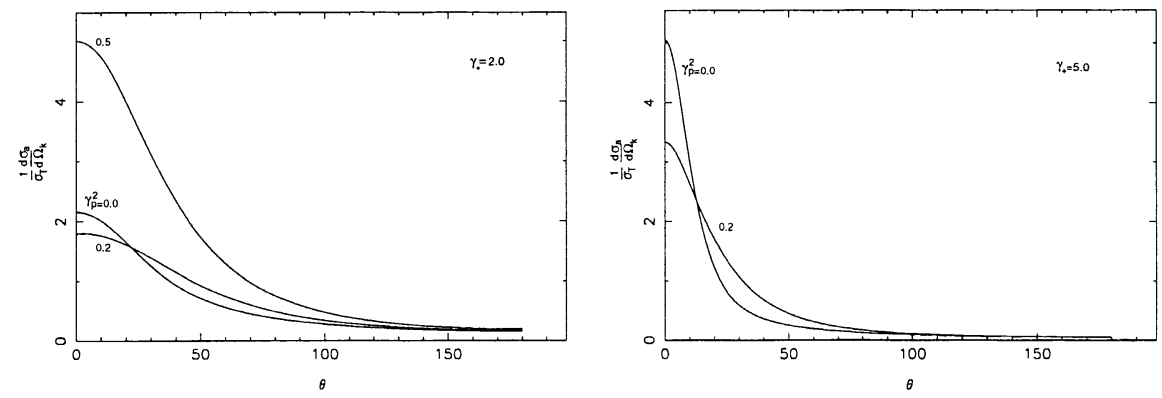

Figure 8. Variation of the differential cross section for pair annihilation with the angle $\theta$ between the positron beam and the photon $\left(\gamma_{1}\right)$ for different positron energies $\gamma_{+}$and plasma density parameters $\gamma_{p}^{2}$.

corresponds to an increase of the frequency of the scattered photon as the plasma density or the parameter $p$ increases, for $\gamma=1$. For $\gamma=10$, the variations with $p$ are very small. The second root also increases with $p$, but lies below the $p=0$ line for small values of scattering angle $\theta$. Further, the quadratic (3) has real roots only over a limited range of scattering angles for a given value of $p$. The variation with $\theta$ of the two differential cross sections corresponding to the two roots (Equation 2) is shown in Figures 4 and 5 for different values of the parameter $p$ and initial photon energy $\gamma$. The cross section corresponding to the first root is found to decrease significantly, with an increase in $p$, for $\theta<90^{\circ}$, and increase for $\theta \geq 90^{\circ}$. For $\gamma=10$, the cross section begins to increase for $\theta \ll 90^{\circ}$. Furthermore, the scattering takes place only for limited values of the scattering angle. The cross section for the second root (Figure 5) shows a similar behavior but becomes very, very large for large scattering angles. This root may not represent the physical situation. 


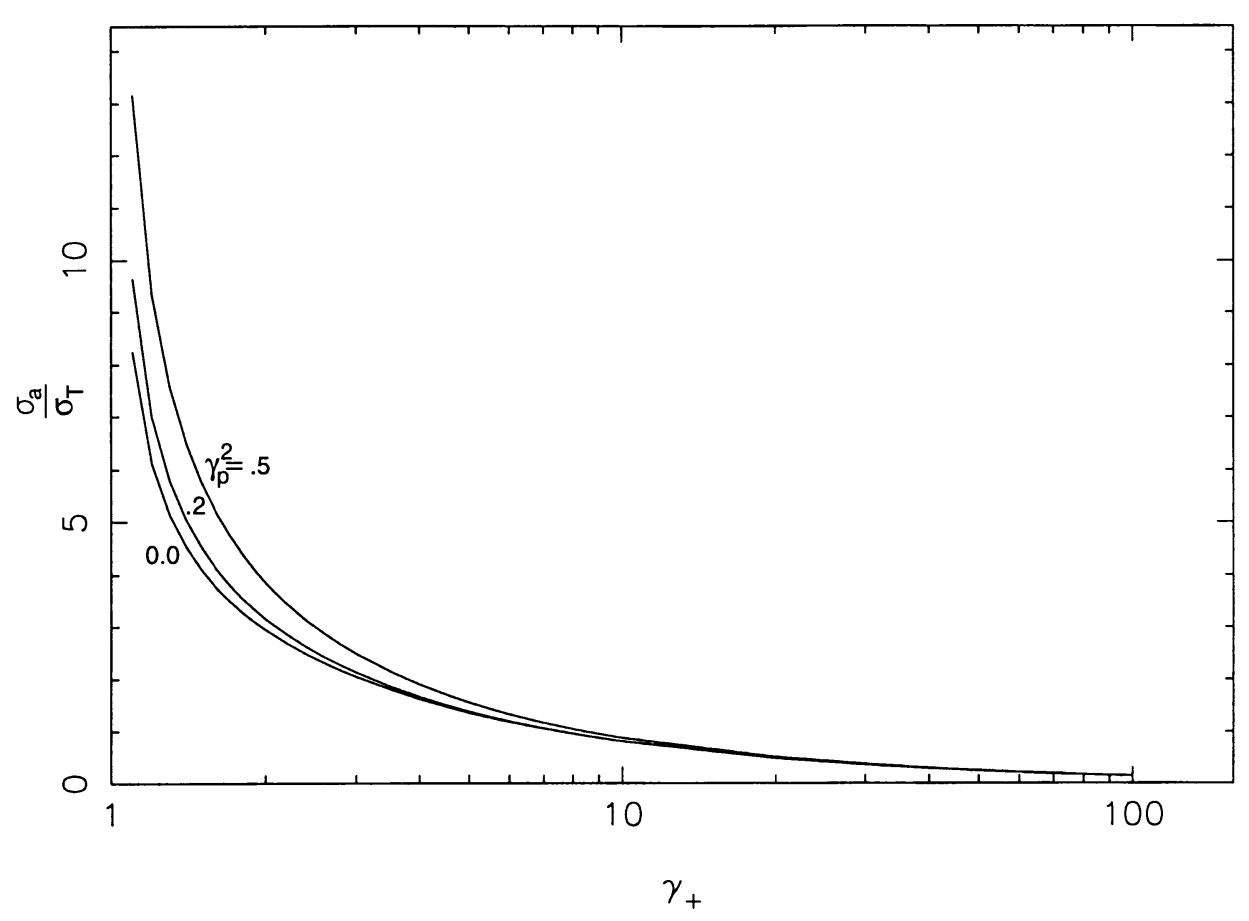

Figure 9. Variation of the total cross section $\sigma_{a}$ for pair annihilation with the positron energy $\gamma_{+}$for different values of the plasma density parameter $\gamma_{p}^{2}$.

\section{Pair Annihilation}

The annihilation of an electron with four-momentum $p_{-}$and spin $S_{-}$, and a positron with four-momentum $p_{+}$and spin $S_{+}$, into two photons with fourmomenta and polarizations $k_{1}, \Lambda_{1}$ and $k_{2}, \Lambda_{2}$ is depicted by the Feynman diagrams in Figure 6 . The similarity with Compton scattering is obvious. By using the crossing symmetry (Reinhardt 1992, 1994), i.e., by replacing $p_{i} \rightarrow p_{-},-p_{f} \rightarrow p_{+},-k \rightarrow k_{1}$, and $k^{\prime} \rightarrow k_{2}$ in the Compton scattering matrix, one can obtain the matrix elements for the pair annihilation process. By appropriately taking care of the phase space factors, averaging over the initial states, and summing over the final states, we find the differential cross section for pair annihilation in the rest frame of the electron to be

$$
\frac{\mathrm{d} \bar{\sigma}_{\mathrm{a}}}{\mathrm{d} \Omega_{\mathrm{k}}} \frac{1}{r_{0}^{2}}=\frac{\left(1+\gamma_{+}\right)\left[\frac{\gamma_{2}}{\gamma_{1}} \frac{1}{A_{1}}+\frac{\gamma_{1}}{\gamma_{2}} \frac{1}{B_{2}}+D \sin ^{2} \theta_{o}+E\right] \sqrt{1-p_{1}}}{2 \beta_{+}\left\{1+\gamma_{+}-\left(1-p_{1}\right)^{-1 / 2} \beta_{+} \cos \theta\right\}\left\{1+\gamma_{+}-\left(1-p_{1}\right)^{1 / 2} \beta_{+} \cos \theta\right\}},
$$



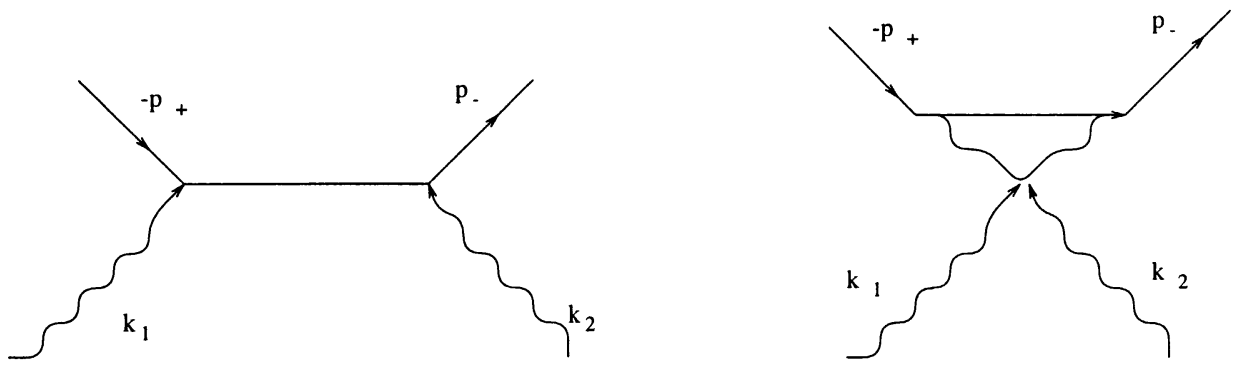

Figure 10. Feynman diagrams for pair production.

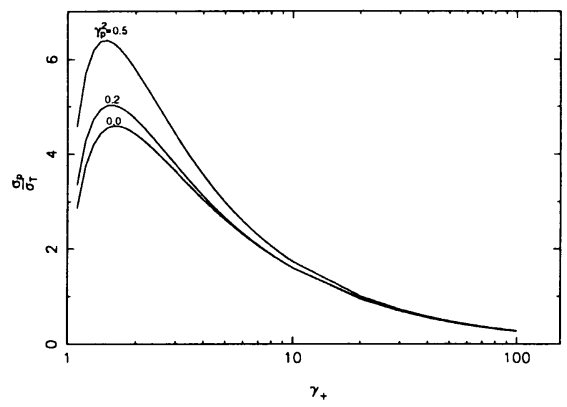

Figure 11. Variation of the pair production cross section $\sigma_{p}$ with positron energy $\gamma_{+}$for different values of the plasma density parameter $\gamma_{p}^{2}$.

where $\gamma_{+} m c^{2}$ is the positron energy, $\gamma_{2}$ and $\gamma_{1}$ are the energies of the two photons, $A_{1}=\left(1-p_{1} \gamma_{1} / 2\right)^{2}, B_{2}=\left(1-p_{2} \gamma_{2} / 2\right)^{2}$,

$$
\begin{gathered}
D=1 / A_{1} B_{2}-\left(1-\sqrt{A_{1} / B_{2}}\right) \gamma_{1}\left(1-p_{1}\right) / 2 A_{1}-\left(1-\sqrt{B_{2} / A_{1}}\right) \gamma_{2}\left(1-p_{2}\right) / 2 B_{2}, \\
E=\left(-p_{1} \gamma_{1} / 2 A_{1} B_{2}\right)\left\{\left(-2 \gamma_{1} / \gamma_{2}\right) \sqrt{A_{1} B_{2}}+A_{1}\left(\gamma_{1}^{2} / \gamma_{2}^{2}-\gamma_{1} / \gamma_{2}\right)-\right. \\
\left.-B_{2}\left(1-\gamma_{2} / \gamma_{1}\right)-2 \sqrt{A_{1} B_{2}}\right\}
\end{gathered}
$$

$$
\begin{gathered}
\sin ^{2} \theta_{o}=\frac{\beta_{+} \sin \theta}{\gamma_{2}\left(1-p_{2}\right)^{1 / 2}}, \quad p_{1}=\gamma_{p}^{2} / \gamma_{1}^{2}, \quad p_{2}=\gamma_{p}^{2} / \gamma_{2}^{2}, \quad \gamma_{p}=\hbar \omega_{p} / m c^{2}, \beta_{+}^{2}=\gamma_{+}^{2}-1, \\
\gamma_{2}=\gamma_{+}+1-\gamma_{1}, \quad \gamma_{1}=\frac{1+\gamma_{+}}{1+\gamma_{+}-\left(\gamma_{+}-t\right) \sqrt{1-p_{1}}}, \quad \text { and } \cos \theta=\frac{\gamma_{+}-\mathrm{t}}{\beta_{+}} .
\end{gathered}
$$

The variation with $\theta$ of the differential cross section for pair annihilation, the angle between the positron beam and the photon $\left(\gamma_{1}\right)$ for different values of the positron energy $\gamma_{+}$, and the plasma density parameter $\gamma_{p}^{2}$ are shown in Figures 7 and 8. Integrating over the solid angle $\mathrm{d} \Omega_{\mathrm{k}}$, the total cross section 
$\sigma_{a}$ for pair annihilation can be determined. The variation of $\sigma_{a}$ with $\gamma_{+}$for different values of $\gamma_{\rho}^{2}$ is shown in Figure 9. It is seen that the cross section for pair annihilation increases with an increase in the plasma density.

\section{Pair Production}

The creation of an electron and a positron by the annihilation of two photons is depicted in Figure 10. Again, using crossing symmetry wherein one replaces $p_{i} \rightarrow-p_{+}, p_{f} \rightarrow p_{-}, k \rightarrow k_{1}$, and $k^{\prime} \rightarrow-k_{2}$ in the Compton scattering matrix, the total cross section for the process of pair production can be determined. More directly, it is known that the total cross section $\sigma_{p}$ for pair production is related to $\sigma_{a}$ (Reinhardt 1992, 1994) as $\sigma_{p}=2\left(\gamma_{+}^{2}-1\right) \sigma_{a} / \gamma_{+}^{2}$. The variation of $\sigma_{p}$ with $\gamma_{+}$for different values of $\gamma_{p}^{2}$ is shown in Figure 11. One can see the threshold behavior of the pair production cross section, as well as its increase with an increase of the plasma density.

The enhancement of the pair annihilation and production cross sections and the decrement of the Compton scattering cross section (for small $\theta$ ) in a plasma are the consequences of the finite mass that a photon acquires in a plasma. Typical plasma densities at which the effects are appreciable are of the order of $10^{30} \mathrm{~cm}^{-3}$. Such a density, for example, exists in the early universe when its temperature was $\sim 10^{10} \mathrm{~K}$. The highly anisotropic nature of Compton scattering with reduced cross sections for forward scattering in a plasma will have important consequences in several astrophysical situations.

Acknowledgments. The author gratefully acknowledges discussions with Dr. R. T. Gangadhara, Dr. P. Ponda, and Mr. B. A. Varghese.

\section{References}

Harris, E. G. 1975, Introduction to Modern Theoretical Physics, Vol. 2 (New York: John Wiley and Sons)

Krishan, V. 1999, Astrophysical Plasmas and Fluids (Dordrecht: Kluwer Academic Publishers)

Landau, L. D. \& Lifshitz, E. M. 1960, Electrodynamics of Continuous Media, trans. J. B. Sykes \& J. S. Bell (Reading: Addison-Wesley)

Reinhardt, G. 1994, Quantum Electrodynamics (Berlin: Springer-Verlag)

Rose, W. K. 1973, Astrophysics (New York: Holt, Rinehart and Winston, Inc.)

Svensson, R. 1990, in NATO ASI Series, Physical Processes in Hot Cosmic Plasmas, eds. B. Wolfgang, A. C. Fabian, \& F. Giovannelli (Dordrecht: Kluwer Academic Publishers), 357 